\title{
Glucose availability impacts proteotoxic stress in Caenorhabditis elegans
}

2 Gatrell, Landon'1, Wilkins, Whitney ${ }^{1}$, Rana, Priya ${ }^{1}$, Farris, Mindy ${ }^{1 *}$

$3 \quad{ }^{1}$ Department of Biology, University of Central Arkansas, Conway, AR, USA

4 * Correspondence:

5 Mindy Farris

$6 \quad$ MFarris@uca.edu

8 Keywords: neurodegeneration $_{1}$, glucose $_{2}, C$. elegans $_{3}$, aging $_{4}$, stress $_{5}$, polyglutamine $_{6}$,

$9 \quad$ Huntington disease ${ }_{7}$. 
Glucose Impacts Polyglutamine Pheirafypes 4.0 International license.

\section{Abstract}

12 Alterations in protein folding may lead to aggregation of misfolded proteins, which is strongly

13 correlated with neurotoxicity and cell death. Protein aggregation has been shown as a normal

14 consequence of aging, but it is largely associated with age-related disease, particularly

15 neurodegenerative diseases like Huntington disease (HD). Huntington disease is caused by a CAG

16 repeat expansion in the huntingtin gene and serves as a useful model for neurodegeneration due to its

17 strictly genetic origin. Research in the model organism Caenorhabditis elegans suggests that glucose

18 protects against cell stress, including proteotoxicity related to aggregation, despite the well-known,

19 lifespan-shortening effects of glucose. We hypothesized that glucose could be beneficial by

20 alleviating energy deficiency, a well-characterized phenomenon in HD, or by upregulating stress

21 resistance pathways. We used C. elegans expressing polyglutamine repeats to quantify lifespan,

22 motility, reproduction, learning, and activity of succinate dehydrogenase (SDH), with and without

23 glucose, to identify the role of glucose in proteotoxicity and neuroprotection. Our data show HD

24 worms on glucose plates exhibited shorter lifespans, no change in motility, learning, or SDH product

25 formation, but had altered reproductive phenotypes similar to dietary restriction. Additionally, worms

26 expressing toxic polyglutamine repeats were unable to learn association of food with a neutral

27 odorant. We also observed tissue-specific differences; polyglutamine appeared to be slightly more

28 toxic to muscle cells than neurons. Rather than increasing energy production, glucose appeared to

29 decrease mitochondrial metabolism, as SDH formation decreases with added glucose. Future work

30 investigating glucose-mediated neuroprotection should focus on connecting metabolism, sirtuin

31 activation, and DAF-16 activation. 
Glucose Impacts Polyglutamine Pheirafypes 4.0 International license.

\section{Introduction}

34 Protein aggregates are a shared phenotype of many fatal neurodegenerative diseases including

35 polyglutamine disorders such as Huntington disease (HD) [1]. The exact nature of cytotoxicity and

36 neuronal death in HD and other neurodegenerative diseases is currently unknown, but many

37 hypotheses have been proposed, including: loss-of-function and/or gain-of-function mutations,

38 excitotoxicity, oxidative stress, aberrant gene expression, and apoptosis [2-5]. HD provides a

39 convenient model for neurodegeneration research, as it is strictly a genetic disease caused by a

40 specific mutation: a CAG trinucleotide repeat expansion in the gene encoding the huntingtin protein

41 (HTT) [6]. HTT is expressed ubiquitously, though most abundantly in neurons, and is found within

42 the nucleus and cytoplasm of the cell. Similarly, HD-afflicted neurons exhibit intranuclear and

43 cytosolic aggregates containing mutant HTT, ubiquitin, and other proteins [7]. What role these

44 aggregates play in neurotoxicity is unclear, with competing hypotheses of deleterious and even

45 protective functions $[8,9]$. Perhaps the most interesting aspect of neurodegenerative disease is the

46 delayed onset of symptoms until later in life, despite lifelong and ubiquitous expression of the mutant

47 protein [10]. This has led to research linking mechanisms of aging to aggregation diseases.

48 Loss of proteostasis is an inherent part of aging in wild-type systems and age-related protein

49 aggregation has been observed in the wild-type strain of the model organism Caenorhabditis elegans

$50 \quad[11,12]$. Down-regulation of proteostasis is of particular importance to neurodegenerative research

51 and is currently hypothesized to be a contributing factor to the age-related progression of

52 neurodegeneration [13]. Another major feature of aging is an individual's interaction with the

53 environment; specifically, diet has been shown to be a major modulator of aging. A common feature

54 of aging research deals with dietary restriction (DR) and glucose enrichment (GE), which lengthen

55 and shorten lifespan respectively. In C. elegans, the transcription factors DAF-16 (dauer abnormal

56 factor 16) and HSF-1 (heat shock factor 1) play critical roles in regulating and effecting these 
Glucose Impacts Polyglutamine Pheirafypes 4.0 International license.

57 phenotypes and both are affected by the activity of the insulin/IGF signaling pathway [14].

58 Upregulating the insulin/IGF pathway via GE results in deactivation of DAF-16 and HSF-1,

59 decreased lifespans, and accelerated aging [14]. Genetic knockdown of DAF-16 and HSF-1 also

60 accelerates aging and animals display aging phenotypes similar to GE [14]. Conversely, the lifespan

61 extension of some DR techniques downregulates the insulin/IGF pathway and requires DAF-16 and

62 HSF-1 [14].

63 Despite shortening lifespan, GE has recently been shown to increase resistance to proteotoxicity in $C$.

64 elegans, as reduced neurodegeneration was seen in worms expressing long polyglutamine tracts

65 (128Q) or TDP-43 when fed high glucose diets [15]. The insulin/IGF pathway appears to be involved

66 in this, as DAF-16 and HSF-1 were required for glucose-mediated neuroprotection [15]. Glucose

67 feeding theoretically increases energy availability, but it is unclear whether this actually leads to

68 increased energy production. Glucose addition has been shown to cause initial increase in

69 mitochondrial function, followed by decrease, while restriction of glucose has been shown to

70 promote mitochondrial metabolism $[16,17]$. It is possible that cells with high glucose diets

71 downregulate energy production pathways to prevent increased ROS accumulation. HTT seems to

72 have an indirect role in energy production in neurons, and HD neurons display many symptoms of

73 energy deficiency (reviewed in [3]). For example, cells expressing a polyglutamine fragment have

74 reduced expression and activity of succinate dehydrogenase (SDH)/Complex II in the electron

75 transport chain [18].

76 To gain a better understanding of the phenotypic effects of glucose-mediated neuroprotection, we

77 asked if glucose enrichment affected phenotypes associated with HD, such as motility and lifespan,

78 in shorter polyglutamine models of C. elegans. Although C. elegans do not contain an ortholog to

79 human HTT, expression of polyglutamine repeats alone has been demonstrated to cause aggregate

80 formation and toxic effects resembling polyglutamine disease phenotypes [19]. We measured 
Glucose Impacts Polyglutamine Pheqiafypes ${ }^{4.0}$ International license.

81 lifespan, motility, reproduction, SDH product formation, and learning in model organisms expressing

82 expanded (40Q), non-toxic (24Q), and threshold (35Q) polyglutamine repeats. We found that while

83 the presence of glucose failed to improve the resistance to proteotoxic stressors for poly-Q worms,

84 through the simultaneous use of multiple different polyglutamine strains of C. elegans, some tissue-

85 specific differences were shown. Glucose effects on reproduction were surprising, as we saw no

86 significant effect of glucose on the brood size or egg-laying pattern of N2 animals, contrary to that

87 seen in studies using lower concentrations of glucose $[14,15]$. Glucose effects on two polyglutamine

88 strains, however, mirror the reduced brood size and delayed egg-laying phenotype of worms under

89 DR conditions [20, 21]. Using a butanone food-association assay, we showed HD worms to be

90 deficient in learning, even as 1-day-old adults, before other proteotoxic phenotypes are apparent.

91 Glucose failed to have a measurable effect on this learning phenotype or on SDH activity.

\section{Materials and Methods}

93 C. elegans Strains

94 Caenorhabditis elegans strains were acquired from the Caenorhabditis Genetics Center at the

95 University of Minnesota. C. elegans strains used in this project were: the N2 Bristol (wild-type), AM101 (rmls 1 10), AM138 (rmls130), AM140 (rmls 132), and AM141 (rmls 133) strains. The polyglutamine strains (AMXXX) express a construct that contains a polyglutamine repeat of varying length tagged by yellow fluorescent protein (YFP), further differentiated by the promotor-driven tissue localization. The AM101 strain has a length of 40Q and the construct is expressed panneuronally. The AM138 strain has a length of 24Q, expressed in the body-wall muscle cells. The

101 AM140 strain has a length of 35Q and is expressed in the body-wall muscle cells; interestingly, the

102 AM140 strain's polyglutamine fragment is on the verge of the toxic threshold and only older worms

103 show aggregation. The AM141 strain has a length of 40Q and is expressed in the body-wall muscle

104 cells. 
Glucose Impacts Polyglutamine Pheirafypes 4.0 International license.

105 Strains were maintained on Nematode Growth Medium (NGM) plates spotted with 250-500 $\mu \mathrm{L}$ of the

106 OP50 strain of E. coli at $20^{\circ} \mathrm{C}$. Prior to experiments, worms were checked under a fluorescent

107 microscope for YFP expression. For experiments, worms were moved onto new NGM plates or

108 NGM plates with 250mM glucose added (referred to as glucose plates, G) as appropriate. When not

109 being manipulated for experiments, worms were kept at $20^{\circ} \mathrm{C}$. Bacterially-starved worms or

110 contaminated plates were not used for experiments.

\section{Lifespan Assay}

112 The lifespans of the C. elegans strains were measured with (G) or without (NGM) 250mM glucose

113 added. Polyglutamine (polyQ) and N2 worms at the larval L4 stage were transferred from general

114 populations to new, clean NGM plates with or without $250 \mathrm{mM}$ glucose $(\mathrm{G})$ spotted with $250 \mu \mathrm{L}$ of

115 the OP50 strain of E. coli. Surviving worms were scored every other day until all experimental

116 worms were dead. Surviving, reproductively active worms were moved to new plates of the same

117 condition to exclude progeny from analyses. Worms were considered dead when they no longer

118 responded to gentle tactile stimulation. Deaths were separated into two major categories: natural and

119 unnatural. Unnatural deaths were accounted for in the survival analysis software.

120 Kaplan-Meier survival curves were generated using JMP software version 12. The log-rank test was

121 used to test for an overall difference between groups at a 0.05 alpha level. The log rank test was also

122 used for pairwise comparisons of interest with a Bonferroni adjusted alpha. There were four

123 comparisons of interest (N2/NGM vs polyQ/NGM; N2/NGM vs N2/glucose; polyQ/NGM vs

124 polyQ/glucose; N2/glucose vs polyQ/glucose) which yielded an adjusted alpha of 0.0125 for pairwise

125 comparisons $(0.05 / 4=0.0125)$.

126 Motility Assay 
Glucose Impacts Polyglutamine Pheqiafypes ${ }^{4.0}$ International license.

127 The movement of $\mathrm{N} 2$ and polyQ worms was measured on plates with (G) or without (NGM) glucose

128 added. Worms at the L4 larval stage were transferred onto new NGM or glucose plates as

129 appropriate. Starting on day 1 of adulthood, the motility of the worms was measured every other day

130 until day 13, at which point worms become predominantly sedentary. Motility was classified as the

131 number of times the tail tip reached the crest of the wave pattern; this frequency was measured by

132 experimenter observation via microscopy for 1 minute. Movements in reverse were also included in

133 data collection. Worms were undisturbed on the plates prior to measurement to relate energy output

134 (motility) to energy availability and were transferred onto new plates post-measurement to exclude

135 progeny. This protocol therefore differed from other protocols that measure paralysis, touch

136 sensitivity, and escape behavior because all of these require experimenter intervention by tactile

137 stimulation.

138 Significance of the effects of the model was tested using a likelihood ratio test with R software

139 version 3.5.1. Pairwise comparisons were tested using simultaneous tests for general linear

140 hypotheses with an adjusted alpha. Data are from 3 separate trials, with $n=10$ worms per strain for

141 each trial.

142 Reproduction Assay

143 The reproduction of individual N2 and polyQ worms was measured on NGM and glucose plates over

144 time. A single worm was placed on an NGM or glucose plate spotted with $250 \mu \mathrm{L}$ of OP50 E. coli.

145 Every day thereafter, the worm was transferred onto a new plate of the same condition. The progeny

146 on the old plates were counted after a hatching period of 24 hours. Only viable progeny were scored

147 for this experiment; unhatched eggs were excluded. This continued until no progeny were found on

148 the plate for 2 consecutive days. Data are from 3 separate trials, with $n=10$ worms per strain for each

149 trial. 
Glucose Impacts Polyglutamine Pheirafypes 4.0 International license.

151 SDH product formation was measured in vitro using a modified version of published methods [18].

152 To extract whole protein, L4 worms matured and reproduced on NGM or glucose plates for 3-4 days,

153 generating a semi-synchronized population. The progeny matured until L4s or young adults were

154 present in high numbers. Worms were washed off the plates using chilled SDH extraction buffer

155 (20mM Tris-HCl, $\mathrm{pH} 7.2 ; 250 \mathrm{mM}$ saccharose; 2mM EGTA; 40mM KCl; 1mg/mL BSA). The worms

156 were gently centrifuged at $82 \mathrm{x}$ g for 1 minute and washed to remove bacteria. Protease inhibitor

157 (5 $\mu \mathrm{L}$ of Thermo-Fisher Halt ${ }^{\mathrm{TM}}$ Protease Inhibitor Single Use Cocktail; 1mM AEBSF, 800nM

158 aprotinin, $50 \mu \mathrm{M}$ bestatin, $20 \mu \mathrm{M}$ leupeptin, $10 \mu$ pepstatin) was added, and then worms were ground

159 on ice for 5 minutes using a tephlon grinder and an overhead stirrer. The lysate was centrifuged at

$160735 \mathrm{x} g$ for 5 minutes to remove cell debris. The concentration of whole protein was measured using

161 the Bradford assay using BSA as a standard.

162 To measure the activity of SDH in the lysate, SDH activity buffer (100mM Tris-HCl, $\mathrm{pH} 8.3 ; 1 \mathrm{mM}$

163 EDTA; 20mM succinate) was added in equal volume to the lysate. A final concentration of $2 \mathrm{mM}$

164 iodonitrotetrazolium chloride was added to start the reaction.

165 Iodonitrotetrazolium (INT) is an electron acceptor and produces a red dye upon reduction. The

166 reaction progressed for approximately 60 minutes at $35^{\circ} \mathrm{C}$ and dye formation was measured using a

167 Nanodrop spectrophotometer at 490nm, with three replicates measured per reaction.

168 SDH product formation was calculated by standardizing the absorbance of INT by the concentration

169 of the lysate. Product formation was analyzed with JMP software version 12 using a two-way

170 ANOVA at a 0.05 alpha level, with Tukey's HSD test for post-hoc analysis.

171 Learning Assay 
Glucose Impacts Polyglutamine Pheqiafypes ${ }^{4.0}$ International license.

172 The ability of N2 and polyQ worms to associate a normally neutral odorant (butanone) to food ( $E$.

173 coli) was measured after exposure to the odorant in the presence of food. Parent worms were

174 transferred to new NGM or glucose plates spotted with $500 \mu \mathrm{L}$ of OP50 E. coli and allowed to

175 reproduce for 8-16 hours, then removed so that progeny were age synchronized. The learning assay

176 was performed according to previously described methods [22].

177 Chemotaxis index was scored by the number of worms at each location and calculated as: $\mathrm{CI}=$

178 (butanone $-\mathrm{EtOH}) /($ total - origin). Statistical significance was analyzed with JMP software version

17912 using a two-way ANOVA.

180 Results

181 Lifespan

182 To determine if glucose is indeed protective against proteotoxicity, the lifespan of wild-type and

183 polyglutamine (polyQ) strains of $C$. elegans were measured under NGM and glucose conditions. If

184 glucose is protective, then the addition of glucose should increase the lifespan of worms expressing

185 toxic polyglutamine repeats (40Q). Since glucose is known to shorten the lifespan of wild-type

186 worms, the lifespans of N2 and of worms expressing non-toxic or threshold polyglutamine repeats

187 (24Q and 35Q) should be shortened compared to their respective NGM lifespans.

188 As expected, the presence of a toxic polyglutamine repeat did shorten the lifespan of C. elegans.

189 Although the lifespan of the pan-neuronal 40Q worms on NGM was not significantly shortened

190 compared to N2 (Figures 1A,B; log-rank test, $\mathrm{p}=0.0137$ ), there was a strong trend toward this. The

191 lifespan of the body-wall muscle 40Q strain on NGM plates was significantly shortened (Figure 1C;

192 log-rank test, $\mathrm{p}<0.0001$ ). The 24Q worms had an increased lifespan on NGM plates compared to

193 N2/wildtype (Figure 1D; log-rank test, $p=0.003$ ), while the 35Q worms exhibited a reduced lifespan

194 on NGM compared to the N2 lifespan (Figure 1E; log-rank test, $\mathrm{p}<0.0001$ ). 
Glucose Impacts Polyglutamine Pheqiofypes 4.0 International license.

195 Fig 1. Glucose did not extend lifespans of transgenic worms expressing polyglutamine constructs.

196 (A) N2 lifespan without (NGM) or with (G) glucose. (B,C,D,E) Lifespans of pan-neuronal 40Q,

197 body-wall muscle 40Q, body-wall muscle 24Q, and body-wall muscle 35Q, respectively, without and

198 with glucose. Averages are shown from three independent experiments for each strain \& condition.

199 Error bars indicate SEM.

200 Exposure to $250 \mathrm{mM}$ glucose shortened the average lifespan of C. elegans by approximately 3-5 days

201 in the N2, 40Q, and 24Q strains (Figures 1A-D). The 35Q strain was the only exception to this; the

202 glucose lifespan was not significantly different than the NGM lifespan (Figure 1E; $\mathrm{p}=0.024$ ).

203 Unexpectedly, the lifespan of toxic polyQ-afflicted worms with glucose was reduced compared to N2

204 worms with glucose. The lifespan of pan-neuronal 40Q worms with glucose was reduced compared

205 to both the same strain on NGM and N2 on glucose (log-rank test, $\mathrm{p}<0.0001$ for both comparisons).

206 The body-wall muscle 40Q on glucose was shorter than the same strain on NGM and N2 on glucose

207 (log-rank test, $\mathrm{p}<0.0001$ for both comparisons). While the 24Q lifespan was shortened with glucose

208 enrichment compared to on NGM, this was not significantly different from the N2/glucose lifespan

209 (log-rank test, $\mathrm{p}<0.0001$ and $\mathrm{p}=0.858$ respectively). Interestingly, the 35Q strain was the only

210 strain that did not exhibit a shorter lifespan on glucose plates (log-rank test, $p=0.0249)$. This

211 lifespan was also not significantly different from the N2/glucose lifespan (log-rank test, $\mathrm{p}=0.9129)$,

212 similar to the 24Q strain. No lifespan-extending, protective effect of glucose was observed in any of

213 the four polyglutamine strains, contrary to expected results.

214 Overall, polyglutamine shortens the lifespan of C. elegans; however, we observed that repeats below

215 the toxic threshold (24Q) increase the lifespan and repeats at the toxic threshold (35Q) decrease

216 lifespan. The addition of glucose shortened the lifespans of the 40Q strains, contrary to expectations.

217 Glucose shortened the lifespan of the N2 and 24Q strains, but not the 35Q strain. The lifespan

218 shortening effect of glucose in the N2 and 24Q strains and the lifespan of the 35Q strain on glucose

219 were not different from each other. 
Glucose Impacts Polyglutamine Pheqiafypes ${ }^{4.0}$ International license.

\section{Motility}

221 The spontaneous movement of wild-type and polyglutamine strains were measured to test the

222 hypothesis that glucose affects polyQ phenotypes based on increased energy availability. Toxic

223 polyQ worms should be less motile than wild-type due to energy deficiency, and glucose enrichment

224 could increase the energy available and provide protection. This assay was designed to observe

225 energy-output and relate output to energy fluctuations; therefore, worms were left undisturbed on the

226 plates, contrary to measurements of the ability to move. This protocol assumed that the amount of

227 spontaneous movement (without experimenter intervention) reflects the abundance of energy

228 available to spend on movement.

229 Spontaneous movement was significantly affected by strain (Figure $2 ; \chi^{2}=103.3$, df $=4, p<0.0001$ ).

230 The N2 strain was more motile than the 40Q, 24Q, and 35Q body-wall muscle strains (Figures 2A,C-

$231 \mathrm{E} ; \mathrm{p}<0.001, \mathrm{p}=0.007, \mathrm{p}<0.0001$, respectively). The pan-neuronal 40Q strain was more motile than

232 all other strains (Figure 2B; $\mathrm{p}<0.0001$ across all comparisons). The motility of 40Q, 24Q, and 35Q

233 body-wall muscle worms was not significantly different across strains.

234 Fig 2. Glucose did not affect motility of transgenic worms expressing polyglutamine constructs. (A)

$235 \mathrm{~N} 2$ motility without (NGM) or with (G) glucose. (B,C,D,E) Motility of pan-neuronal 40Q, body-wall 236 muscle 40Q, body-wall muscle 24Q, and body-wall muscle 35Q, respectively, without and with

237 glucose. Averages are shown from three independent experiments for each strain \& condition. Error

238 bars indicate SEM.

239 Spontaneous movement was not significantly impacted by glucose $\left(\chi^{2}=1.05, \mathrm{df}=1, \mathrm{p}=0.305\right)$, but

240 tended to decrease motility early in adulthood, especially in the N2 strain (Figure 2).

\section{Reproduction}

242 To further test how glucose affects high energy functions, the reproduction patterns of the wild-type

243 and toxic polyQ (40Q) strains were measured. If polyglutamine proteotoxicity results in energy

244 deficiency and this deficiency disrupts high energy functions, then polyglutamine worms should

245 show decreased fecundity compared to N2. Furthermore, if neuroprotection occurs through increased 
Glucose Impacts Polyglutamine Pheqiafypes ${ }^{4.0}$ International license.

246 availability of energy, then the presence of glucose should rescue reproductive deficiency in the 40Q

247 strains.

248 The wild-type strain of C. elegans lays many eggs over a relatively short period of time ( $\sim 300$ over 7

249 days, Figure 3A). The addition of a toxic polyglutamine repeat, regardless of localization, decreases

250 the brood size (Figures 3B,C). Contrary to expectations, the addition of glucose decreased the brood

251 size in both 40Q strains (Figure 3). However, glucose also increased the reproductive lifespan of the

252 worms, as worms on glucose plates continued to lay eggs for 1-2 days longer than worms on NGM

253 plates, similar to effects of dietary restriction $[20,21]$.

254 Fig 3. Glucose altered the egg-laying phenotype of transgenic worms expressing toxic polyglutamine 255 constructs. (A) N2 reproduction without (NGM) or with (G) glucose. (B,C) Reproduction of pan256 neuronal 40Q and body-wall muscle 40Q, respectively, without and with glucose. Averages are 257 shown from three independent experiments for each strain \& condition. Error bars indicate SEM.

\section{SDH Product Formation}

259 SDH product formation was measured in young adult worms to observe how glucose affects energy

260 deficiency in HD. We predicted the 40Q strains would show decreased SDH product formation

261 compared to $\mathrm{N} 2$ due to the presence of toxic polyQ repeats. The addition of glucose was expected to

262 increase SDH product formation based on the increased availability of energy in the N2, 24Q, and

263 35Q strains. If SDH deficiency in HD is the result of direct action of polyglutamine on SDH, then

264 SDH deficiency in 40Q worms should persist despite glucose. However, if SDH deficiency is the

265 result of energy depletion and lack of substrate, then increased glucose intake should increase SDH

266 product formation.

267 SDH product formation seemed to vary widely between strains and conditions, though there was a

268 detectable overall significant difference between groups (Figure 4; two-way ANOVA, F9,20 = 2.79, p

$269=0.026)$. The strain effect was the only significant effect in the model $\left(\mathrm{F}_{4}=3.53, \mathrm{p}=0.024\right)$,

270 although the effect of glucose verged on significance $\left(F_{1}=3.55, p=0.074\right)$ with no interaction effect 
Glucose Impacts Polyglutamine Pheqiafypes ${ }^{4.0}$ International license.

$271\left(\mathrm{~F}_{4}=1.86, \mathrm{p}=0.15\right)$. Between strains, the product formation in 35Q worms was higher than in 40Q

272 worms expressing the polyQ in the same tissue $(\mathrm{p}=0.016)$. This was the only statistically significant

273 pairwise comparison in the model.

274 Fig 4. SDH product formation only changes upon addition of glucose for the non-toxic

275 polyglutamine construct. Averages are shown from three independent experiments for each strain \&

276 condition. Error bars indicate SEM.

277 Although not statistically significant, some interesting trends can be seen in the data. The SDH

278 product formation of pan-neuronal 40Q on NGM was not different from N2 on NGM. The 24Q and

27935 Q worms both had increased activity on NGM compared to all other strains, despite both strains

280 carrying a non-toxic repeat. Glucose tended to decrease SDH product formation in the N2 and 24Q

281 strains, but not in the 35Q or 40Q strains. The SDH product formation of the pan-neuronal 40Q strain

282 actually tended to increase with glucose addition, while the enzyme activity of body-wall muscle

283 40Q and 35Q strains remained the same.

\section{Learning}

285 As shown previously, the N2 strain of C. elegans is able to learn an artificial association between

286 food (E. coli) and a neutral odorant (butanone) (Figure 5). Interestingly, in the pan-neuronal 40Q

287 strain, learning was completely abolished, indicating a novel early-life (1-day-old adult) polyQ

288 phenotype (two-way ANOVA, $\mathrm{F}_{1}=126.1, \mathrm{p}<0.0001$ ). The addition of glucose had no impact on

289 learning in either strain $\left(\mathrm{F}_{1}=0.131, \mathrm{p}=0.72\right)$.

290 Fig 5. Pan-neuronal toxic polyglutamine worms show no ability to associate food with an odorant, 291 even early in life. Glucose has no effect on the ability of these or wild-type animals to learn food

292 association. Averages are shown from three independent experiments for each strain \& condition.

293 Error bars indicate SEM.

\section{Discussion}

295 The aim of this project was to assess the glucose-dependent stress resistance of non-disease-

296 associated (24Q), threshold (35Q), and expanded (40Q) polyglutamine models of Caenorhabditis 
Glucose Impacts Polyglutamine Pheqiafypes ${ }^{4.0}$ International license.

elegans. To test the hypothesis that glucose is protective against proteotoxic stress, four strains of

polyglutamine-expressing worms were studied under normal and high glucose diets, along with the

wild-type/N2 strain. Worms with toxic polyQ expansions were expected to be deficient in lifespan,

motility, reproduction, SDH product formation, and learning phenotypes compared to the wild-type

strain. If glucose was protective, then high glucose diets should have improved or rescued deficiency.

302 Furthermore, if glucose was protective based upon increased energy availability, then SDH product

303 formation should have correlated with increased lifespan, motility, etc.

304 The pan-neuronal 40Q strain failed to associate butanone with E. coli (Figure 5; p < 0.0001). One

305 mechanism of synaptic plasticity and learning is long-term potentiation, which functions through

306 NMDA receptors [23]. Stimulation of these receptors recruits AMPA receptors to the post-synaptic

307 membrane, modulates the excitability of the post-synaptic neuron, and strengthens the connection

308 [23]. Several studies using mouse-models of HD and post-mortem human brains have shown that HD

309 presents several dysfunctions with NMDA receptor function and AMPA receptors (extensively

310 reviewed in $[24,25])$. In C. elegans, NMDA and AMPA receptor homologs are present, and the

311 worm can learn via long-term potentiation [26, 27]. The fact that pan-neuronal 40Q worms

312 performed poorly in learning tasks suggests that polyQ disrupts long-term potentiation in C. elegans,

313 similar to mouse models of HD.

314 NMDA and AMPA receptors are glutamate-stimulated calcium channels, known to be deficient in

$315 \mathrm{HD}$, and are heavily implicated in the excitotoxicity model of neurodegeneration [28, 24]. Calcium is

316 an important intracellular signal and functions to control mitochondrial activity, apoptotic signaling,

317 neurotransmitter release, etc. [29, 30]. If NMDA and AMPA receptors are deficient in C. elegans,

318 then aberrant glutamate signaling may lead to calcium influxes in neuronal circuits and

319 excitotoxicity. Calcium influx, therefore, should increase energy production, SDH activity, and ROS

320 production, although SDH is not activated directly by $\mathrm{Ca}^{2+}[30]$. However, SDH product formation 
Glucose Impacts Polyglutamine Pheirafypes 4.0 International license.

321 was not different in the pan-neuronal 40Q strain compared to the N2 strain (Figure 4). One

322 explanation for this could be that increased ROS production negatively regulated or reduced activity

323 of SDH before product formation was measured [31].

324 Increased calcium influxes may also increase acetylcholine signaling at the neuromuscular junction,

325 leading to increased motility in the pan-neuronal 40Q strain (Figure 2; $p=0.0001$ ). Interestingly, this

326 effect seems to be time-dependent; increased motility in this strain appears to be restricted to early in

327 life, although this was not tested statistically. Aberrant calcium signaling is unlikely to affect the

328 motility of the body-wall muscle 40Q strain because expression of the polyQ is restricted to muscles.

329 Neurons of the body-wall muscle 40Q strain should fire normally, and thus the incoming signal at the

330 neuromuscular junction should resemble that of wild-type. Dysfunction in the body-wall muscle 40Q

331 strain should originate in the muscle and this is supported by the motility of the body-wall muscle

332 40Q strain, which was decreased compared to the pan-neuronal 40Q and N2 strains (Figure 2; p $<$

3330.0001 for both comparisons). The increase in motility in the pan-neuronal 40Q strain and the

334 decrease in motility in the body-wall muscle 40Q strain suggest that proteotoxicity manifests

335 differently in neurons than in muscles.

336 The tissue-specific effects of polyglutamine extended to other phenotypes: lifespan of the body-wall

337 muscle 40Q strain was slightly shorter than that of the pan-neuronal 40Q strain (Figure 1), though

338 this effect was not statistically significant. Motility in all three muscular polyglutamine strains was

339 decreased when compared to the pan-neuronal strain (Figure 2; $<<0.0001$ for all comparisons).

340 Brood size was slightly decreased in body-wall muscle 40Q compared to pan-neuronal 40Q (Figure

3413 3). SDH product formation was slightly decreased in polyQ muscles compared to wild-type, but not

342 neurons (Figure 4). Considering that both the pan-neuronal and body-wall muscle 40Q strains

343 express a repeat of the same length, these data suggest that muscles are slightly more vulnerable to

344 polyglutamine than neurons. 
Glucose Impacts Polyglutamine Pheqiafypes ${ }^{4.0}$ International license.

345 Differences in vulnerability may be due to a fundamental difference in the chaperone activity in

346 muscles and neurons, which was investigated in C. elegans under heat stress [32]. Heat stress causes

347 proteins to misfold; cells resist protein misfolding via the heat shock response and upregulation of

348 molecular chaperones [32]. Under the control of HSF-1, muscles are more resistant to heat stress,

349 while neurons showed a stronger recovery from heat stress. In older worms, neurons show more

350 resistance but no recovery (similar to young muscles), while older muscles lose resistance and

351 recovery altogether [32]. What this implies for chronic proteotoxic stress is unclear, but it could

352 provide support for increased sensitivity to polyglutamine in muscles, leading to shorter lifespans and

353 more deficiency. Although degeneration of specific populations of neurons is generally thought to be

354 causative of the specific phenotypes associated with the various polyglutamine diseases, some

355 research indicates that dysfunction is not exclusive to neurons and may explain some of the motor

356 deficits in HD (reviewed in [33-40]).

357 Curiously, the 24Q strain was no more motile than the 35Q and 40Q body-wall muscle strains, which

358 are at or above the toxic threshold (Figure $2 ; \mathrm{p}=0.29, \mathrm{p}=0.71$ respectively). This suggests that the

359 presence of polyQ in muscle cells, no matter the degree of toxicity, reduces movement in C. elegans.

360 The 35Q strain was another peculiarity in the data; this was the only strain whose lifespan was not

361 shortened by glucose (Figure $1 ; p=0.0249$ ). Surprisingly, the 24Q strain lived significantly longer

362 than the $\mathrm{N} 2$ strain (Figure $1 ; \mathrm{p}=0.003$ ). One potential and interesting explanation for this is that the

363 repeat may induce cellular stress that the cell is easily able to manage via activation of stress

364 resistance pathways, thus providing a net stress resistant phenotype (hormesis). If the 24Q strain is

365 receiving a hormetic benefit from its non-toxic polyQ repeat, then DAF-16 and/or HSF-1 could be

366 active in this strain under NGM conditions and may be the hormetic effector(s). One result of DAF-

36716 activation is stimulation of metabolism, and 24Q's increased SDH product formation (not

368 significant) supports the hypothesis that DAF-16 is active (Figure 4; [41-43]). In mammals, the DAF- 
Glucose Impacts Polyglutamine Pheqiafypes ${ }^{4.0}$ International license.

36916 homolog FOXO3a has been found to have increased nuclear localization in mouse HD cell lines

370 [44]. FOXO3 has been shown to differentially regulate transcription in HD, connected to altered

371 sleep and stress phenotypes appearing as early HD symptoms [45]. The mechanism associated with

372 these early, non-motor symptoms may also be connected to the failure we observed of young (1-day-

373 old) 40 Q worms in the butanone learning assay.

374 SDH deficiency has been well characterized in HD models, but its origin is unclear [18, 3]. One

375 potential explanation is that the direct action of polyglutamine affects SDH via gain of function

376 action. SDH product formation in the body-wall muscle 40Q strain was not significantly reduced

377 compared to wild-type, nor in the pan-neuronal 40Q strain (Figure 4). This alone suggests that

378 polyglutamine itself is not the effector of SDH deficiency. Furthermore, glucose enrichment was not

379 indicative of increased energy production, at least in terms of SDH, so SDH deficiency is unaffected

380 by increased substrate availability. These results suggest that SDH deficiency arises from another

381 source altogether. Interestingly, enzymes of the TCA cycle, such as aconitase, are particularly

382 sensitive to ROS, which is increased in HD [3]. However, the ETC is the major generator of ROS in

383 the cell, and the results of this study did not show increased activity of SDH/complex II in toxic

384 polyQ strains when compared to N2. Alternatively, the subunits of SDH function separately in the

385 TCA cycle and ETC, meaning that SDH product formation may not be indicative of complex II

386 activity [31].

387 Collectively, these data make clear that glucose, at the relatively high $(250 \mathrm{mM})$ concentrations used

388 in this study, is not protective in the polyglutamine models we examined. The glucose concentrations

389 used here are possibly beyond the threshold of protection, which may explain why we did not see the

390 protection observed in other studies, which also used longer polyglutamine constructs [15]. Glucose-

391 mediated neuroprotection was previously found to be DAF-16-dependent and DAF-16 does have

392 some control over metabolism [46, 15]. However, the results of this study support other studies that 
Glucose Impacts Polyglutamine Pheirafypes 4.0 International license.

393 show glucose may not increase mitochondrial energy production, as SDH product formation was not

394 stimulated by glucose enrichment (Figure 4; [16, 17]). Glucose-mediated neuroprotection may be due

395 to DAF-16 activity, which would lead to interesting future questions, including what is activating

396 DAF-16, and how the downstream targets of DAF-16 interact with factors effecting polyglutamine

397 proteotoxicity.

\section{Conclusions}

399 This study assessed the glucose-dependent stress resistance of Caenorhabditis elegans in response to 400 polyglutamine proteotoxicity. As neurodegeneration poses a significant health risk, identification of

401 both neuroprotective and neurotoxic factors is essential. C. elegans expressing toxic polyglutamine

402 repeats exhibited shorter lifespans and increased deficiency, as measured by lifespan, motility,

403 reproduction, SDH product formation, and learning. Glucose $(250 \mathrm{mM})$ did not improve these deficits

404 in 24Q, 35Q, and 40Q polyglutamine models, and exacerbated decrease in lifespan. This glucose

405 concentration is likely beyond a threshold of activation of protective mechanisms, dependent on

$406 \mathrm{NAD}^{+}$levels, sirtuin activation, and DAF-16 dependent gene expression.

\section{Conflict of Interest}

408 The authors declare that the research was conducted in the absence of any commercial or financial

409 relationships that could be construed as a potential conflict of interest.

\section{Acknowledgments}

411 The authors would like to thank the support of the University Research Council, University of

412 Central Arkansas, AR. We acknowledge funding from The Arkansas IDeA Network of Biomedical

413 Research Excellence (Arkansas INBRE).

\section{Author Contributions}


Glucose Impacts Polyglutamine Pheirafypes 4.0 International license.

415 LG performed all lifespan, motility, and SDH assay experiments. LG and MF performed the

416 reproduction experiments. WW and PR performed the learning assay experiments. MF supervised the

417 project. LG and MF wrote the manuscript with input from all authors.

418

419

420

421

422

423

424

425

426

427

428

429

430

431

432

433

434

435

436

437

438

439

440

441

442

443

444

445

446

447

448

449

450

451

452

453

454

455

456

\section{References}

1. Ross AR, Poirier MA. Protein aggregation and neurodegenerative disease. Nature Medicine. 2004; 10, S10-S17.

2. Cuervo AM, Stefanis L, Fredenburg R, Lansbury PT, Sulzer D. Impaired degradation of mutant $\alpha-$ synuclein by chaperone-mediated autophagy. Science. 2004; 305, 1292-1295.

3. Mochel F, Haller R. Energy deficient in Huntington disease: why it matters. Journal of Clinical Investigation. 2011; 121, 493-499.

4. Rego AC, Oliveira CR. Mitochondrial dysfunction and reactive oxygen species in excitotoxicity and apoptosis: implications for the pathogenesis of neurodegenerative diseases.

Neurochemical Research. 2003; 28, 1563-1574.

5. Saura CA, Choi SY, Beglopoulos V, Malkani S, Zhang D, Shankaranarayana BS, et al. Loss of presenilin function causes impairments of memory and synaptic plasticity followed by agedependent neurodegeneration. Neuron. 2004; 42, 23-36.

6. Huntington's Disease Collaborative Research Group, The. A novel gene containing a trinucleotide repeat that is expanded and unstable on Huntington's disease chromosomes. Cell. 1993; 72, 971-983.

7. Morton AJ, Lagan MA, Skepper JN, Dunnett SB. Progressive formation of inclusions in the striatum and hippocampus of mice transgenic for the human Huntington's disease mutation. Journal of Neurocytology. 2000; 20, 679-702.

8. Jackson WS, Tallaksen-Greene SJ, Albin RL, Detloff PJ. Nucleocytoplasmic transport signals affect the age at onset of abnormalities in knock-in mice expressing polyglutamine within an ectopic protein context. Human Molecular Genetics. 2003; 12, 1621-1629.

9. Ross AR, Poirier MA. What is the role of protein aggregation in neurodegeneration? Nature Reviews. 2005; 6, 891-897.

10. Bhide PG, Day M, Sapp E, Schwarz C, Sheth A, Kim J, et al. Expression of normal and mutant huntingtin in the developing brain. Journal of Neuroscience. 1996; 16, 5523-5535.

11. Koga H, Kaushik S, Cuervo AM. Protein homeostasis and aging, the importance of exquisite quality control. Aging Research Reviews. 2011; 10, 205-215.

12. David DC, Ollikainen N, Trinidad JC, Cary MP, Burlingame AL, Kenyon C. Widespread protein aggregation as an inherent part of aging in C. elegans. PLoS Biol. 2010 ; 8, e1000450.

13. Hartl FU, Bracher A, Hayer-Hartl M. Molecular chaperones in protein folding and proteostasis. Nature. 2011; 475, 324-333.

14. Lee SJ, Murphy CT, Kenyon C. Glucose shortens lifespan of C. elegans by downregulating DAF16/FOXO activity and aquaporin gene activation. Cell Metabolism. 2009; 10, 379-391.

15. Tauffenberger A, Vaccaro A, Aulas A, Vande Velde C, Parker JA. Glucose delays age-dependent proteotoxicity. Aging Cell. 2012; 11, 856-866.

16. Russell JW, Golovoy D, Vincent AM, Mahendru P, Olzmann JA, Mentzer A, et al. High glucoseinduced oxidative stress and mitochondrial dysfunction in neurons. FASEB Journal. 2002; 13,1738-1748. 
Glucose Impacts Polyglutamine Pheirafypes 4.0 International license.

17. Schulz TJ, Zarse K, Voigt A, Urban N, Birringer M, Ristow M. Glucose restriction extends Caenorhabditis elegans life span by inducing mitochondrial respiration and increasing oxidative stress. Cell Metabolism. 2007; 6, 280-293.

18. Benchoua A, Trioulier Y, Zala D, Gaillard MC, Lefort N, Dufour N. Involvement of mitochondrial complex II defects in neuronal death produced by N-terminal fragment of mutated huntingtin. Molecular Biology of the Cell. 2006; 17, 1653-1663.

19. Satyal SH, Schmidt E, Kitagawa K, Sondheimer N, Lindquist S, Kramer JM, et al. Polyglutamine aggregates alter protein folding homeostasis in Caenorhabditis elegans. Proc Natl Acad Sci U $S$ A. 2000; 97, 5750-5755.

20. Szewczyk NJ, Udranszky IA, Kozak E, Sunga J, Kim SK, Jacobson LA, et al. Delayed development and lifespan extension as features of metabolic lifestyle alteration in C. elegans under dietary restriction. J Exp Biol. 2006; 209, 4129-4139.

21. Crawford D, Libina N, Kenyon C. Caenorhabditis elegans integrates food and reproductive signals in lifespan determination. Aging Cell. 2007; 6, 715-721.

22. Kauffman AL, Ashraf JM, Corces-Zimmerman MR, Landis JN, Murphy CT. Insulin signaling and dietary restriction differentially influence the decline of learning and memory with age. PLoS Biology. 2010; 8, e1000372.

23. Lynch MA. Long-term potentiation and memory. Physiology Reviews. 2004; 84, 87-136.

24. Fan MM, Raymond LA. N-methyl-D-aspartate (NMDA) receptor function and excitotoxicity in Huntington's disease. Progress in Neurobiology. 2007; 81, 272-293.

25. Mandal M, Wei J, Zhong P, Cheng J, Duffney LJ, Liu W, et al. Impaired AMPA receptor trafficking and function by mutant huntingtin. Journal of Biological Chemistry. 2011; 286, 33719-33728.

26. Kano T, Brockie PJ, Sassa T, Fujimoto H, Kawahara Y, Iino Y, et al. Memory in Caenorhabditis elegans is mediated by NMDA-type ionotropic glutamate receptors. Curr Biol. 2008; 18, 1010-1015.

27. Rose JK, Kaun KR, Chen SH, Rankin CH. GLR-1, a non-NMDA glutamate receptor homolog, is critical for long-term memory in Caenorhabditis elegans. Journal of Neuroscience. 2003; 23, 9595-9599.

28. Dong XX, Wang Y, Qin ZH. Molecular mechanisms of excitotoxicity and their relevance to pathogenesis of neurodegenerative diseases. Acta Pharmacologica Sinica. 2009; 30, 379-387.

29. Contreras L, Drago I, Zampese E, Pozzan T. Mitochondria, The calcium connection. Biochimica et Biophysica Acta. 2010; 1797, 607-618.

30. Denton RM. Regulation of mitochondrial dehydrogenases by calcium ions. Biochimica et Biophysica Acta. 2009; 1787, 1309-1316.

31. Zorov DB, Juhaszova M, Sollott SJ. Mitochondrial reactive oxygen species (ROS) and ROSinduced ROS release. Physiological Reviews. 2014; 94, 909-950.

32. Kern A, Ackermann B, Clement AM, Duerk H, Behl C. HSF1-controlled and age-associated chaperone capacity in neurons and muscle cells of C. elegans. PLoS One. 2010; 5, e8568.

33. Bradford JW, Li S, Li XJ. Polyglutamine toxicity in non-neuronal cells. Cell Research. 2010; 20, 400-407.

34. Carter RJ, Lione LA, Humby T, Mangiarini L, Mahal A, Bates GP, et al. Characterization of progressive motor deficits in mice transgenic for the human Huntington's disease mutation. Journal of Neuroscience. 1999; 19, 3248-3257.

35. Chaturvedi RK, Adhihetty P, Shukla S, Hennessy T, Calingasan N, Yang L, et al. Impaired PGC1alpha function in muscle in Huntington's disease. Human Molecular Genetics. 2009; 18, 3048-3065. 
Glucose Impacts Polyglutamine Pheráfype's.0 International license.

36. Luthi-Carter R, Hanson SA, Strand AD, Bergstrom DA, Chun W, Peters NL, et al. Dysregulation of gene expression in the R6/2 model of polyglutamine disease: parallel changes in muscle and brain. Human Molecular Genetics. 2002; 11, 1911-1926.

37. Ribchester RR, Thomson D, Wood NI, Hinks T, Gillingwater TH, Wishart TM, et al. Progressive abnormalities in skeletal muscle and neuromuscular junctions of transgenic mice expressing the Huntington's disease mutation. European Journal of Neuroscience. 2004; 20, 3092-3114.

38. Sathasivam K, Hobbs C, Turmaine M, Mangiarini L, Mahal A, Bertaux F, et al. Formation of polyglutamine inclusions in non-CNS tissue. Human Molecular Genetics. 1999; 8, 813-822.

39. Strand AD, Aragaki AK, Shaw D, Bird T, Holton J, Turner C, et al. Gene expression in Huntington's disease skeletal muscle, a potential biomarker. Human Molecular Genetics. 2005; 14, 1863-1876.

40. Weydt P, Pineda VV, Torrence AE, Libby RT, Satterfield TF, Lazarowski ER, et al. Thermoregulatory and metabolic defects in Huntington's disease transgenic mice implicate PGC-1alpha in Huntington's disease neurodegeneration. Cell Metabolism. 2006; 4, 349-362.

41. Jacobs KM, Pennington JD, Bisht KS, Aykin-Burns N, Kim HS, Mishra M, et al. SIRT3 interacts with the DAF-16 homolog FOXO3a in the mitochondria, as well as increases FOXO3a dependent gene expression. International Journal of Biological Sciences. 2008; 4, 291-299.

42. Lee SS, Kennedy S, Tolonen AC, Ruvkun G. DAF-16 target genes that control C. elegans lifespan and metabolism. Science. 2003; 300, 644-647.

43. McElwee J, Bubb K, Thomas JH. Transcriptional outputs of the Caenorhabditis elegans forkhead protein DAF-16. Aging Cell. 2003; 2, 111-121.

44. Kannike K, Sepp M, Zuccato C, Cattaneo E, Timmusk T. Forkhead transcription factor FOXO3a levels are increased in Huntington disease because of overactivated positive autofeedback loop. J Biol Chem. 2014; 289, 32845-32857.

45. Scarpa JR, Jiang P, Losic B, Readhead B, Gao VD, Dudley JT, et al. Systems genetic analyses highlight a TGF $\beta$-FOXO3 dependent striatal astrocyte network conserved across species and associated with stress, sleep, and Huntington's Disease. PLoS Genet. 2016; 12, e1006137.

46. Artal-Sanz M, Tavernarakis N. Mechanisms of aging and energy metabolism in Caenorhabditis elegans. Life. 2008; 60, 315-322. 

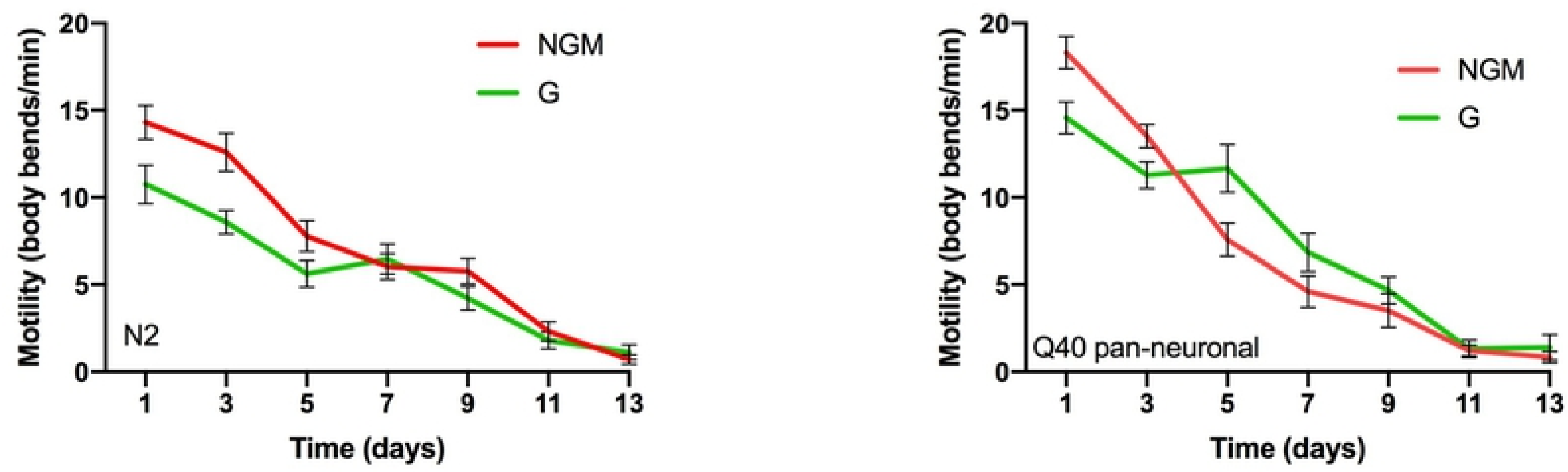

bioRxiv preprint doi: https://doi.org/10.1101/763060; this version posted September 9, 2019. The copyright holder for this preprint (which was not certified by peer review) is the author/funder, who has granted bioRxiv a license to display the preprint in perpetuity. It is made available under aCC-BY 4.0 International license.

C
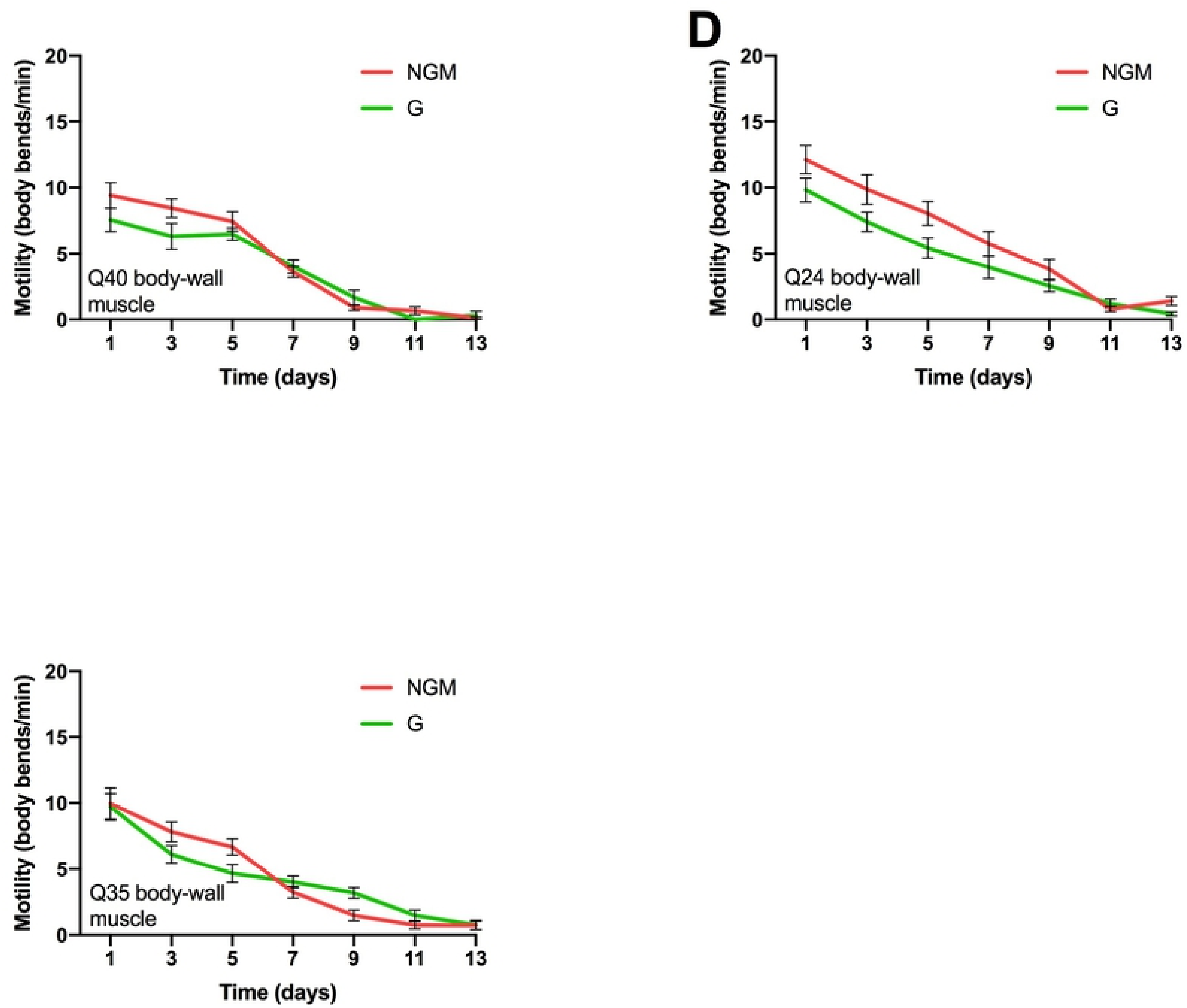


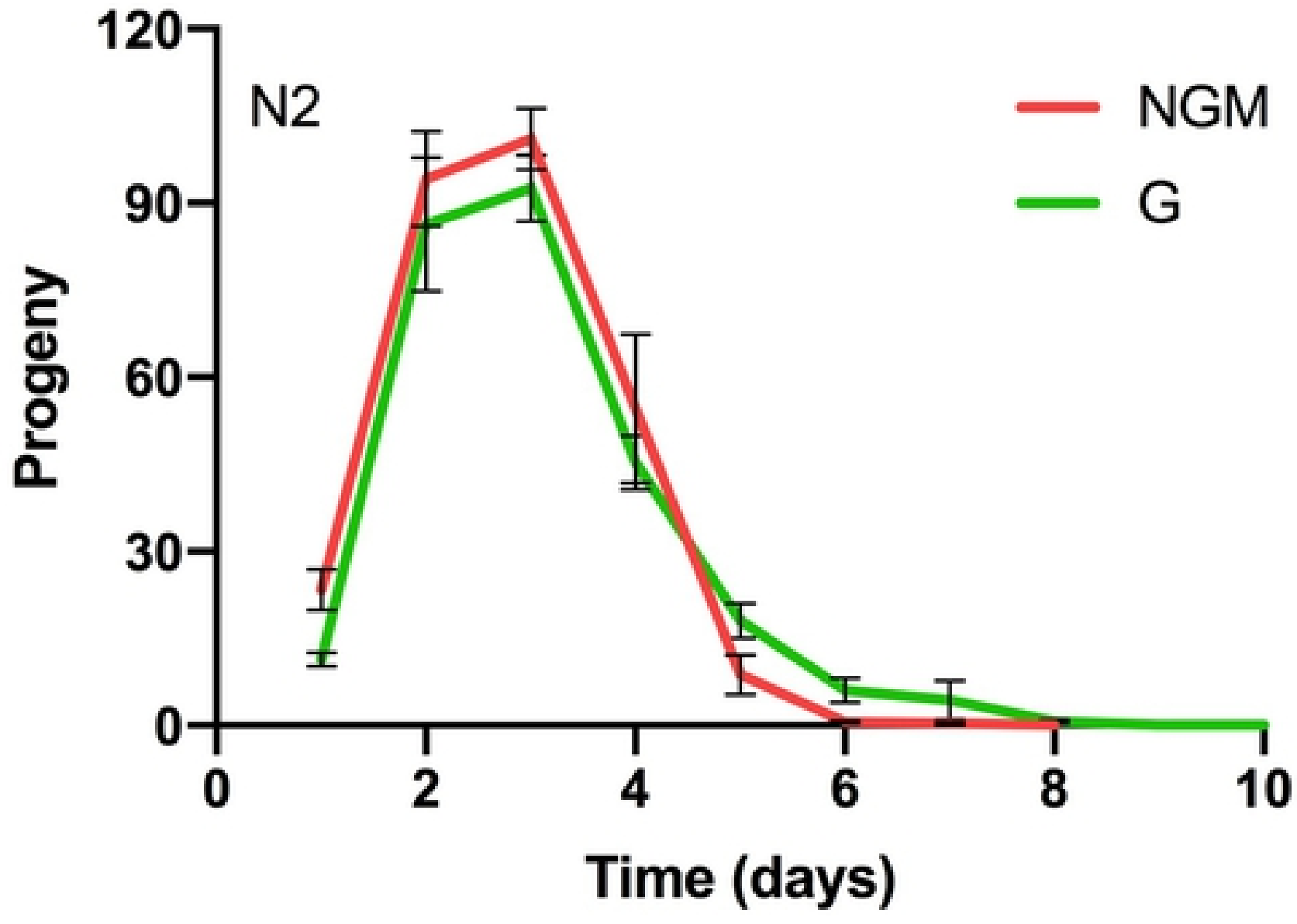

bioRxiv preprint doi: https://doi.org/10.1101/763060; this version posted September 9, 2019. The copyright holder for this preprint (which was not certified by peer review) is the author/funder, who has granted bioRxiv a license to display the preprint in perpetuity. It is made available

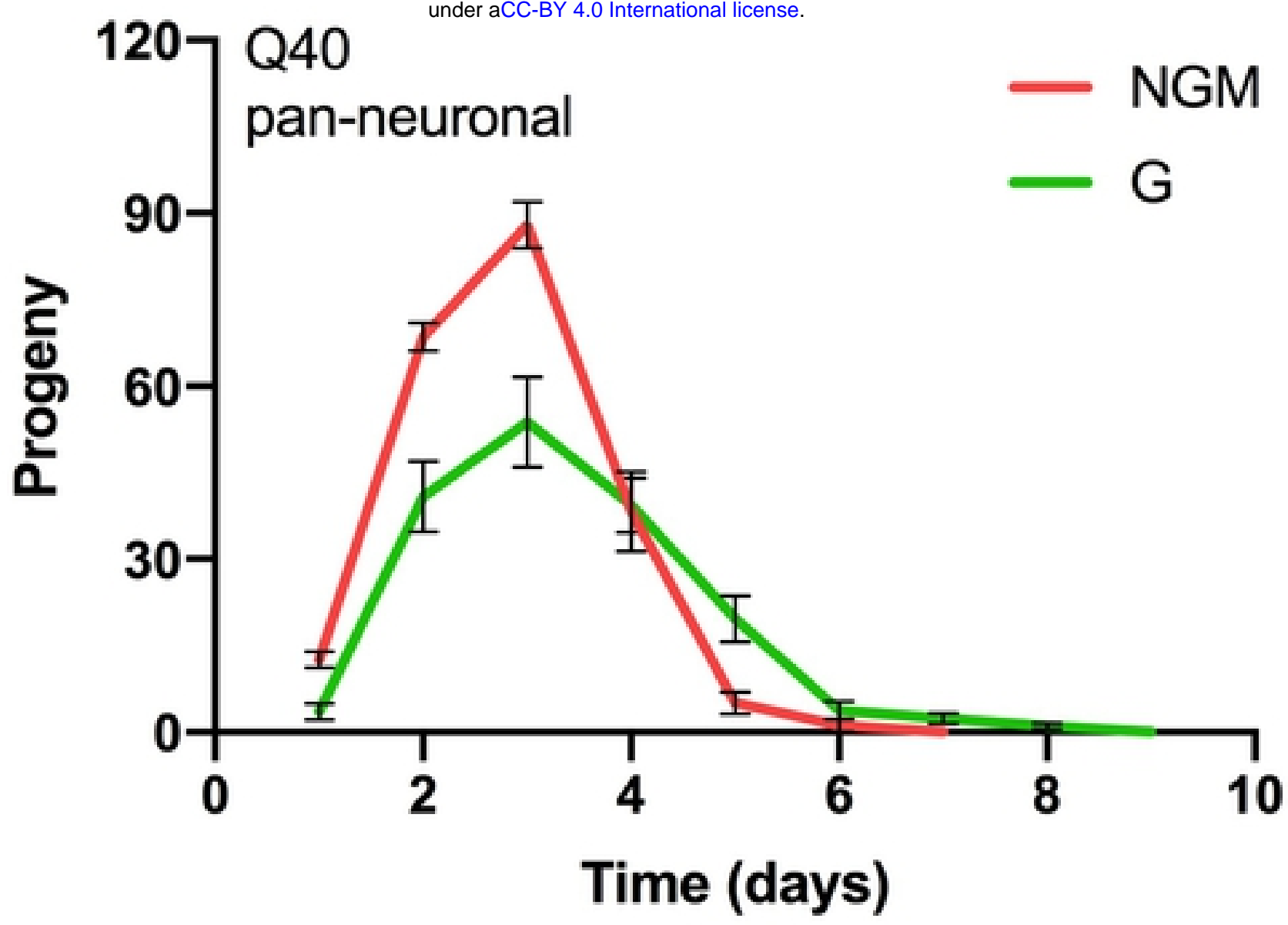

C

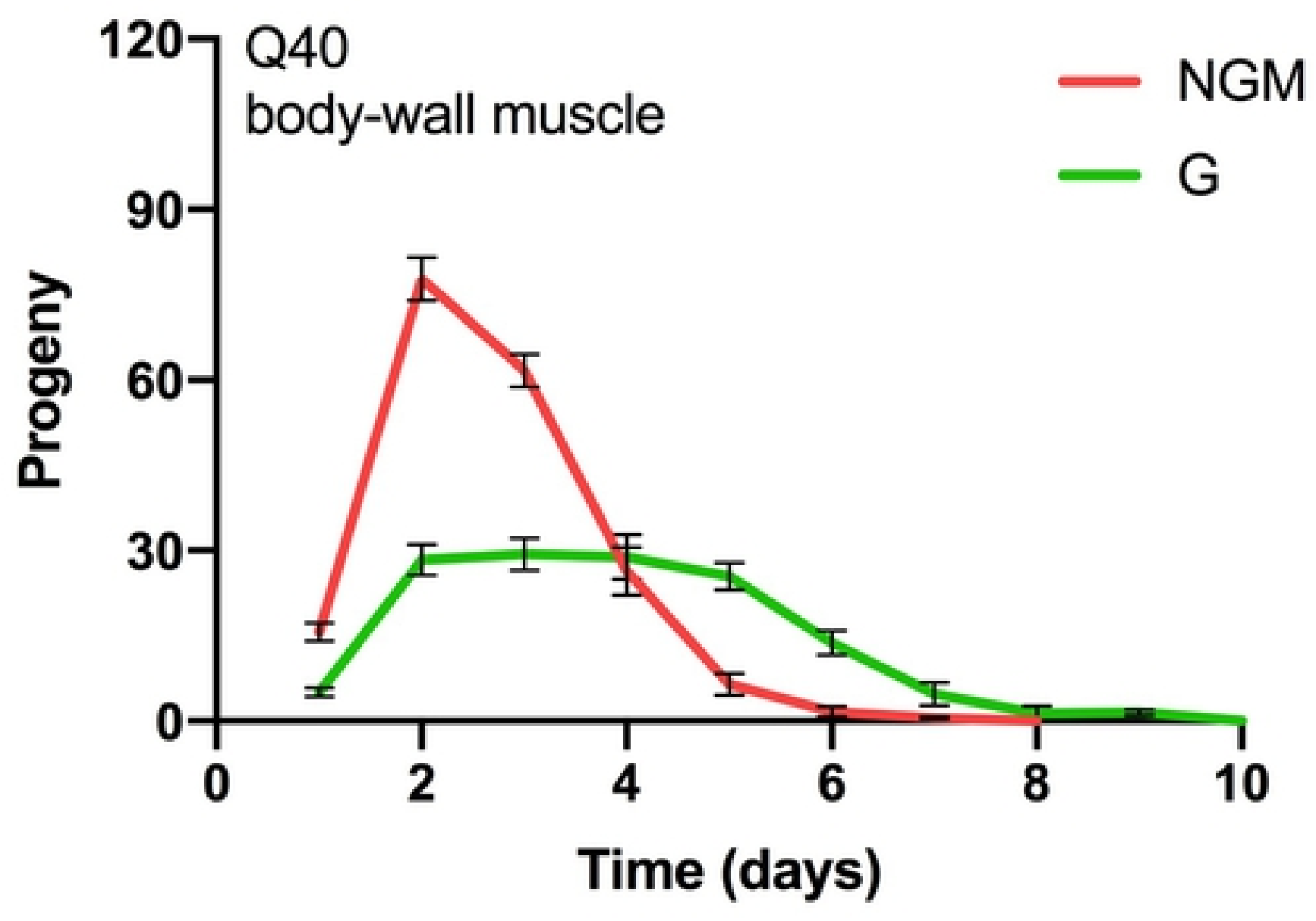




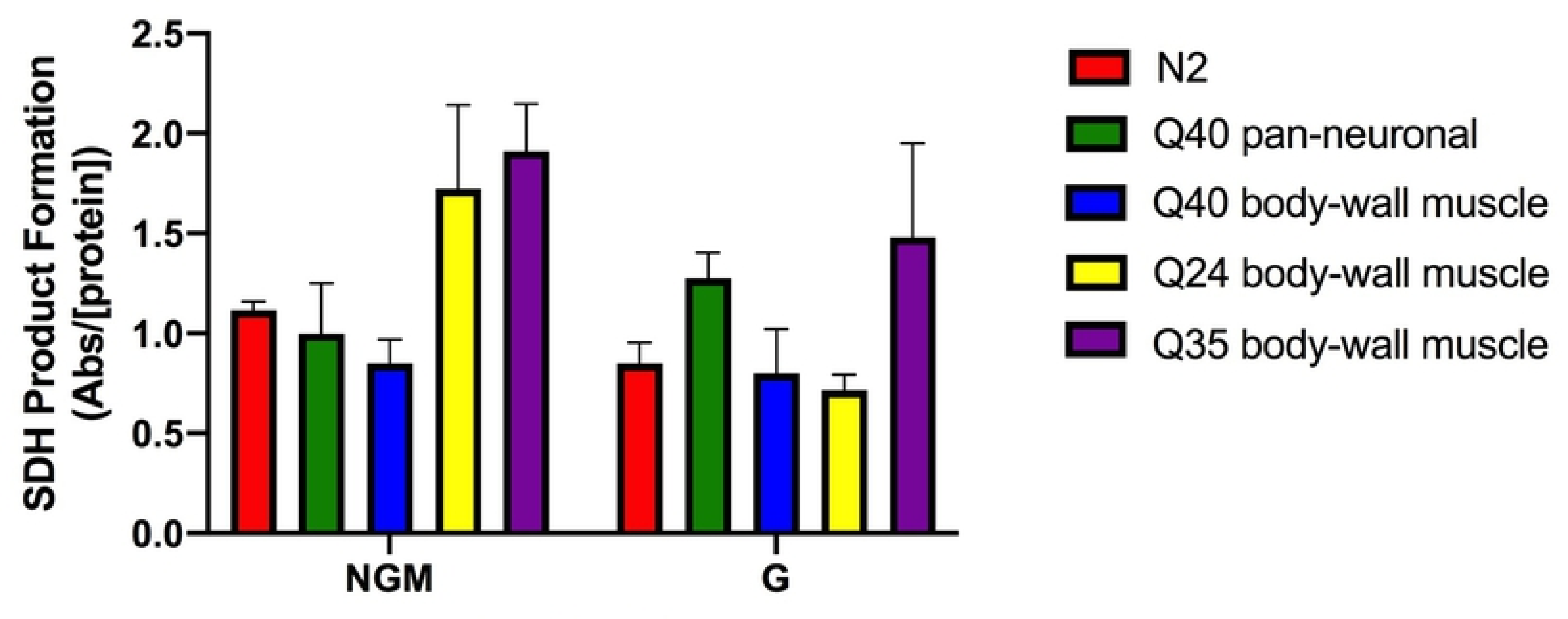

Treatment 


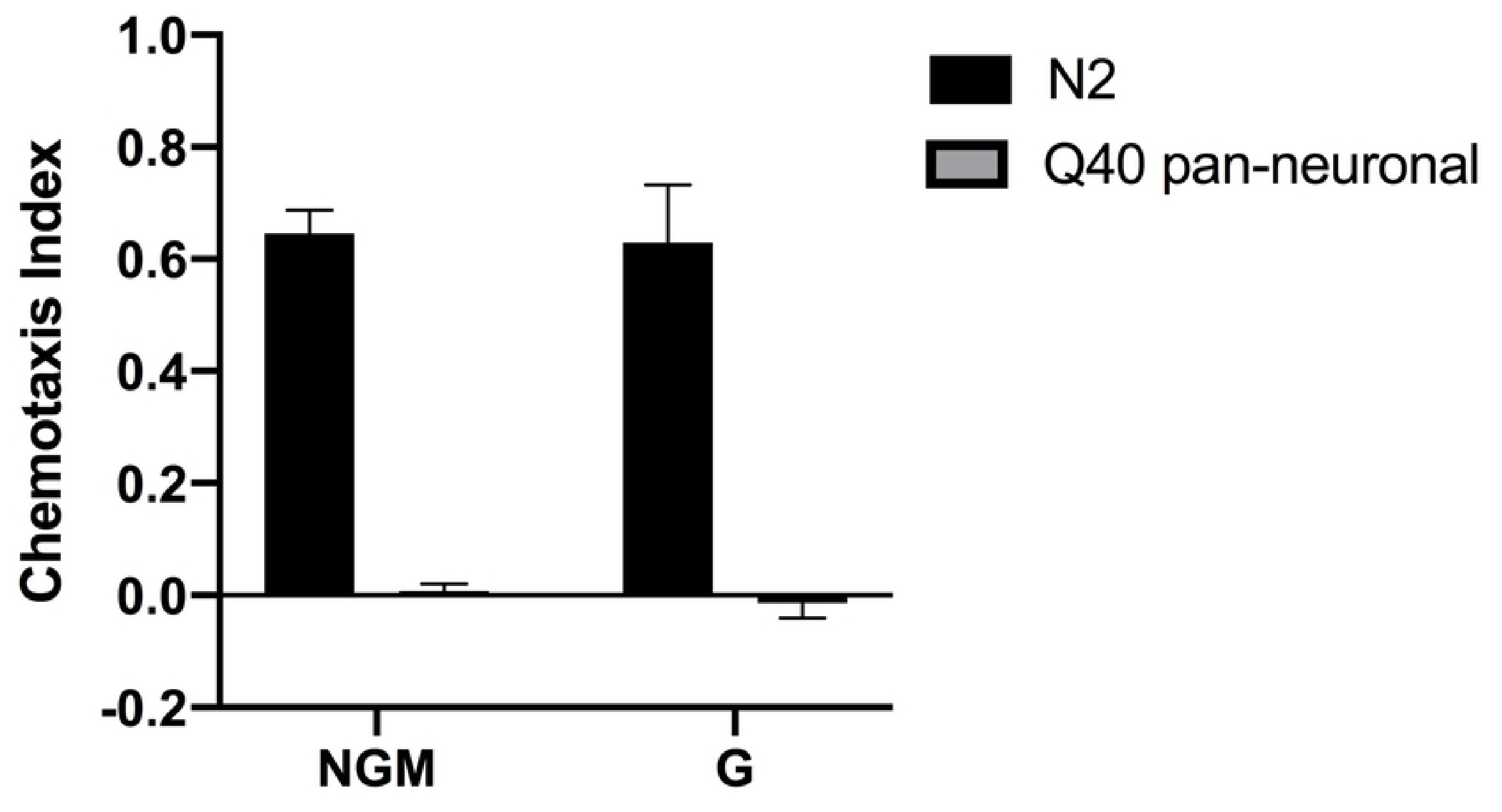

Treatment 